\title{
BMJ Open Association between statin use and herpes zoster: systematic review and meta-analysis
}

\author{
Lailai Fan, ${ }^{1}$ Yangyang Wang, ${ }^{2}$ Xiang Liu, ${ }^{1}$ Xueqiang Guan ${ }^{3}$
}

To cite: Fan L, Wang Y, Liu X, et al. Association between statin use and herpes zoster: systematic review and meta-analysis. BMJ Open 2019;9:e022897. doi:10.1136/ bmjopen-2018-022897

- Prepublication history and additional material for this paper are available online. To view these files, please visit the journal online (http://dx.doi. org/10.1136/bmjopen-2018022897).

Received 13 March 2018 Revised 6 August 2018 Accepted 30 November 2018

Check for updates

(C) Author(s) (or their employer(s)) 2019. Re-use permitted under CC BY-NC. No commercial re-use. See rights and permissions. Published by BMJ.

${ }^{1}$ Department of Urology, The Second Affiliated Hospital and Yuying Children's Hospital of Wenzhou Medical University, Wenzhou, China

${ }^{2}$ Department of Rehabilitation, The Second Affiliated Hospital and Yuying Children's Hospital of Wenzhou Medical University, Wenzhou, China

${ }^{3}$ Department of Cardiology, The Second Affiliated Hospital and Yuying Children's Hospital of Wenzhou Medical University, Wenzhou, China

Correspondence to

Dr Xueqiang Guan;

wzsgxq@163.com

\begin{abstract}
Objective Statins are commonly prescribed worldwide. In addition to being potent lipid-lowering agents, statins have immunomodulating properties that may increase the risk of varicella zoster virus reactivation. This adverse effect may have substantial public health implications. Design We performed a meta-analysis of observational studies to assess the association between statin use and the risk of herpes zoster infection. We searched PubMed, Embase, Web of Science and Cochrane databases to identify studies published from 1980 to 2018. The multivariate-adjusted ORs were pooled using randomeffect models, and subgroup and sensitivity analyses were performed to examine the source of heterogeneity.

Result Six studies were analysed, with a total of more than two million participants. We determined if the use of statins might increase the risk of infection of herpes zoster (OR 1.18, 95\% Cl 1.11 to 1. 25). We detected significant heterogeneity $\left(I^{2}=91.2 \% ; p<0.000\right)$, and determined that the heterogeneity arises from regional differences.

Conclusion The use of statins may increase the risk of herpes zoster infection. Because the studies included are limited and there may be potential bias, further studies are warranted.
\end{abstract}

\section{INTRODUCTION}

Statins (3-hydroxy-3-methylglutaryl-coenzyme A reductase inhibitors) are used to lower levels of low-density lipoprotein cholesterol, and are widely prescribed for the prevention of cardiovascular diseases (CVD). ${ }^{12}$ In addition to acting as potent lipid-lowering agents, statins have immunomodulating properties that may increase the risk of infectious diseases. $^{34}$

Herpes zoster occurs as a reactivation of a latent infection with varicella zoster virus (VZV), causing postherpetic neuralgia and acute and chronic pain. ${ }^{5}$ The pain caused by inflammation and neuronal destruction can be disruptive to daily activities, decreasing quality of life for the elderly. ${ }^{6}$ As statins are commonly prescribed worldwide, if statin use increases the risk of VZV reactivation, this adverse effect may present substantial public health implications.

\section{Strengths and limitations of this study}

We performed a comprehensive systematic search for eligible studies. This is the first systematic review and meta-analysis to specifically evaluate the association between statin use and the risk of herpes zoster.

- The studies included in our meta-analysis included many participants with long-term follow-up time. Literature eligibility was assessed by two investigators independently. No significant publication bias was found.

- This was a meta-analysis of observational studies, allowing the conclusion that an association exists, but this type of analysis cannot determine if a causal relationship exists.

- We found significant heterogeneity across studies, due to regional differences. The study by Chung et al contributed much heterogeneity, but the result remained significant after exclusion of this study.

- We did not study the effects of different statins on the risk of herpes zoster due to insufficient data. Some results might be influenced by the conversion of other measures to $\mathrm{OR}$.

Many epidemiological studies have reported an association between statin use and the risk of herpes zoster, ${ }^{7-12}$ but there has been no meta-analysis to systematically evaluate all available data. To address this need, we conducted a comprehensive systematic review and meta-analysis of observational studies to assess the association between statin use and the risk of herpes zoster.

\section{METHODS}

\section{Search strategy}

We followed the guidelines described in the Meta-Analysis of Observational Studies in Epidemiology. ${ }^{13}$ We first systematically searched PubMed and Embase databases on 20 December 2015 for studies of the association between statins and herpes zoster. To ensure our study included all available up-to-date results, we systematically did an 
additional database search of PubMed, Embase, Web of Science and Cochrane on 20 July 2018. We also checked the references of included studies and reviews. The search focused on statins and herpes zoster as subject terms and keywords. The detailed search strategies are shown in the online supplementary material 1.

\section{Study selection}

Two investigators independently assessed literature eligibility, and disagreements were settled by discussion and consensus. Articles were included in the systematic review if: (1) the authors reported data from an original, peer-reviewed study (ie, not review articles or conference abstracts); (2) the study was a cohort study or case-control study (case reports were not included due to a lower quality of evidence-based medical evidence); and (3) the authors reported risk estimates of herpes zoster among users of statins. For studies that resulted in multiple publications, we included the article with the longest follow-up time or that presented the most incident cases. We identified articles that qualified for further examination by performing an initial screen of identified titles and abstracts, followed by a full-text review.

\section{Data extraction}

Two investigators independently extracted the following information from the studies: authors, publication year, study region, study design, risk estimates (95\% CI), patient mean age, follow-up time or study period, female percentage, diagnosis of herpes zoster, assessment of the use of statins, types of statins used, number of study participants (number of case participants and control participants for case-control studies, and the number of exposures and non-exposures for cohort studies) and confounder adjustment. If any information was unavailable or not clear from a published report, we collected the relevant data by directly corresponding with the authors.

We used the Newcastle-Ottawa Quality Assessment Scale $^{14}$ to evaluate the quality of each study with consideration of selection, comparability and exposure for non-randomised studies. ${ }^{15}$ For the case-control and cohort studies, different evaluation criteria were used. A total score of less than or equal to 3 was considered poor quality, a score of 4-6 was considered moderate quality and a score of 7-9 was deemed high quality. Poor quality studies were excluded in the sensitivity analysis. The details of study assessment are presented in online supplementary material 2.

\section{Data synthesis and analysis}

The fully adjusted risk estimates were used to estimate the association between statin use and the risk of herpes zoster. The HR and relative ratio values were regarded equivalent to the OR for the low incidence of diseases. Forest plots were made to visually assess the ORs and the corresponding $95 \% \mathrm{CI}$ across studies. The heterogeneity across studies was assessed by the Cochrane $Q$ statistic (using a significance level of $\mathrm{p}<0.10$ ) and the $\mathrm{I}^{2}$ statistic (this parameter ranges from $0 \%$ to $100 \%$ with lower values corresponding to less heterogeneity). ${ }^{16}$ The ORs were pooled using the DerSimonian and Laird inversevariance-weighted random-effect models. ${ }^{17}$

The influences of participant characteristics on the results were assessed by subgroup analyses of study type, study region, number of study participants, female percentage, mean age and the quality of the study. We omitted each study individually to test the influence of studies on the heterogeneity and the robustness of the analysis. ${ }^{18}$ The potential publication bias was examined by visual inspection of the funnel plot and the Egger's test result $(\mathrm{p}<0.10) .{ }^{19}$

The analyses were performed with STATA V.14.1 (Stata Corp). A $p$ value $<0.05$ was considered statistically significant, except where otherwise specified. ${ }^{20}$

\section{Patient and public involvement}

No patients and/or the general public were involved in this study.

\section{RESULTS}

\section{Literature search}

Two investigators independently assessed study eligibility and performed data extraction. The results were compared, discussed, and consensus was reached. The initial search yielded 249 articles. Among these, 38 duplicate articles were identified. An additional study was identified by a search of Google Scholar using search terms stains and herpes zoster. After the first round of screening based on titles and abstracts, 15 articles were retained and subjected to further review. After detailed examination, nine articles were excluded. ${ }^{21-29}$ Seven studies were excluded because they were reviews, one study was excluded because it was a conference abstract and one study was excluded because it was a letter. Ultimately, we included six articles ${ }^{7-12}$ in the meta-analysis (figure 1).

\section{Study characteristics}

The characteristics of the included six studies are presented in table 1 . The six studies were published between 2008 and 2018. Two studies were evaluated as high quality, ${ }^{112}$ and the rest were of moderate quality; no study was excluded for poor quality. Three studies were performed in Asia, ${ }^{811}$ two in North America ${ }^{710}$ and one in Europe ${ }^{12}$ The participants of one study were limited to veterans. ${ }^{10}$ Follow-up duration ranged from 1.95 to 11 years, with a median of 5.9 years. The mean age of study participants was between 51.7-73 years, and the participants included in one study were all older than $66 .{ }^{7}$ The largest study ${ }^{12}$ included 694295 participants, and the smallest study ${ }^{10}$ included 18951 participants. All studies had roughly a $50 \%$ sex ratio except one study with only 3.3 per cent women. ${ }^{10}$ Three studies ${ }^{711} 12$ included seven types of statins, two studies ${ }^{89}$ included six types of statins and one study ${ }^{10}$ included five types of statins. In the included studies, the diagnosis of herpes zoster all relied 
The related literatures were obtained from the database $(n=249$, pubmed $=24$, embase $=172$, web of science $=50$, cochrane $=3$ )
Get related literature by other resources $(n=1)$

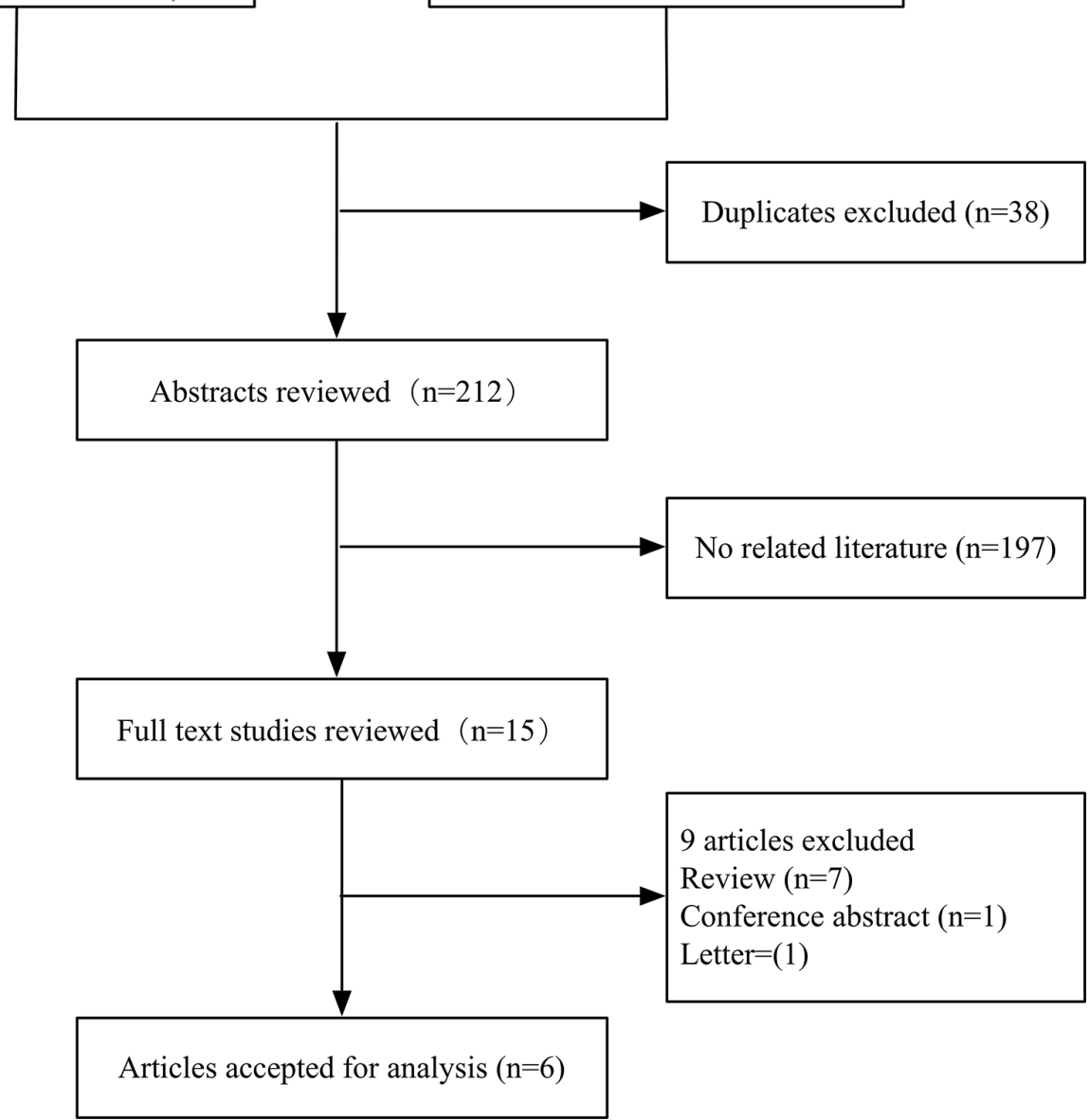

Figure 1 Flow chart of the meta-analysis of association between statin use and herpes zoster.

on the International Classification of Diseases code. The assessment of stain use relied on prescriptions or medication records. The adjustment for potential confounding factors differed between studies, and most risk estimates were adjusted for age and gender.

\section{Statins and herpes zoster}

The majority of studies reported a positive association; only one study reported an OR value that was not statistically different than $1.00 .^{10}$ Three studies reported doseresponse analysis, ${ }^{7812}$ but the data could not be used in a dose-response meta-analysis due to different definitions of dose in the different studies. Two of these studies, Matthews et $a l^{12}$ (low dose, OR 1.12 (95\% CI 1.10 to 1.14 ); medium dose, OR 1.15 (95\% CI 1.11 to 1.18 ); high dose, OR 1.26 (95\% CI 1.15 to 1.39$)$ ) and Chen $e$ t $a l^{8}$ (low dose, OR 0.80 (95\% CI 0.76 to 0.85 ); high dose, OR $2.96(95 \%$ CI 2.74 to 3.20$)$ ) showed a higher risk of herpes zoster infection in patients that received a higher dose. Antoniou et $a l^{7}$ (medium dose, OR 1.05 (95\% CI 1.00 to 1.10); high dose, OR 1.03 (95\% CI 0.90 to 1.19)) reported no appreciable difference in the risk of herpes zoster with stain dose. $^{7}$ Two studies ${ }^{89}$ reported separate OR calculations for men and women, and one study reported an OR value for male participants that was not statistically significant. The pooled OR from a random-effect model of women was 1.32 (95\% CI 1.27 to $1.37, \mathrm{I}^{2}=0 \%$ ), and the pooled OR from a random-effect model of men was $1.17(95 \%$ CI 1.05 to $1.31, \mathrm{I}^{2}=77 \%$ ). Both male and female patients exhibited a higher risk of herpes zoster, with a slightly higher risk for women. This result needs to be further verified due to the small number of study participants.

The adjusted OR $(95 \% \mathrm{CI})$ value related to herpes zoster was determined as 1.18 (95\% CI 1.11 to 1.25 , figure 2). Additionally, we detected an obvious heterogeneity $\left(\mathrm{I}^{2}=91.2 \% ; \mathrm{p}<0.000\right)$.

\section{Publication bias}

There was no publication bias based on the visual inspection of the funnel plot (figure 3) and the result of Egger's test $(\mathrm{p}=0.646)$.

\section{Subgroup and sensitivity analyses}

We conducted subgroup analyses to try to identify the sources of heterogeneity. Analyses were performed by study type, study region, percentage of female, the 


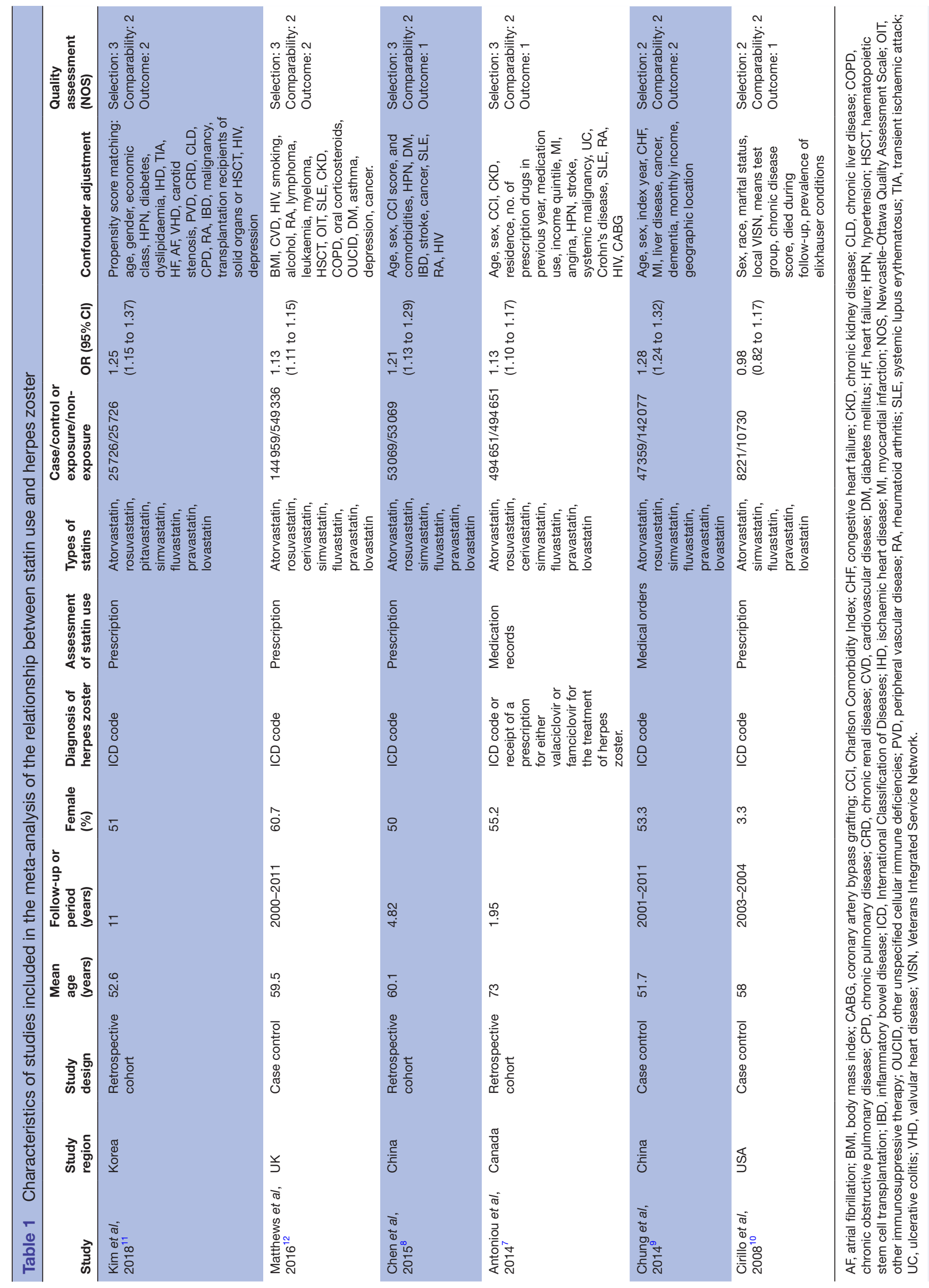




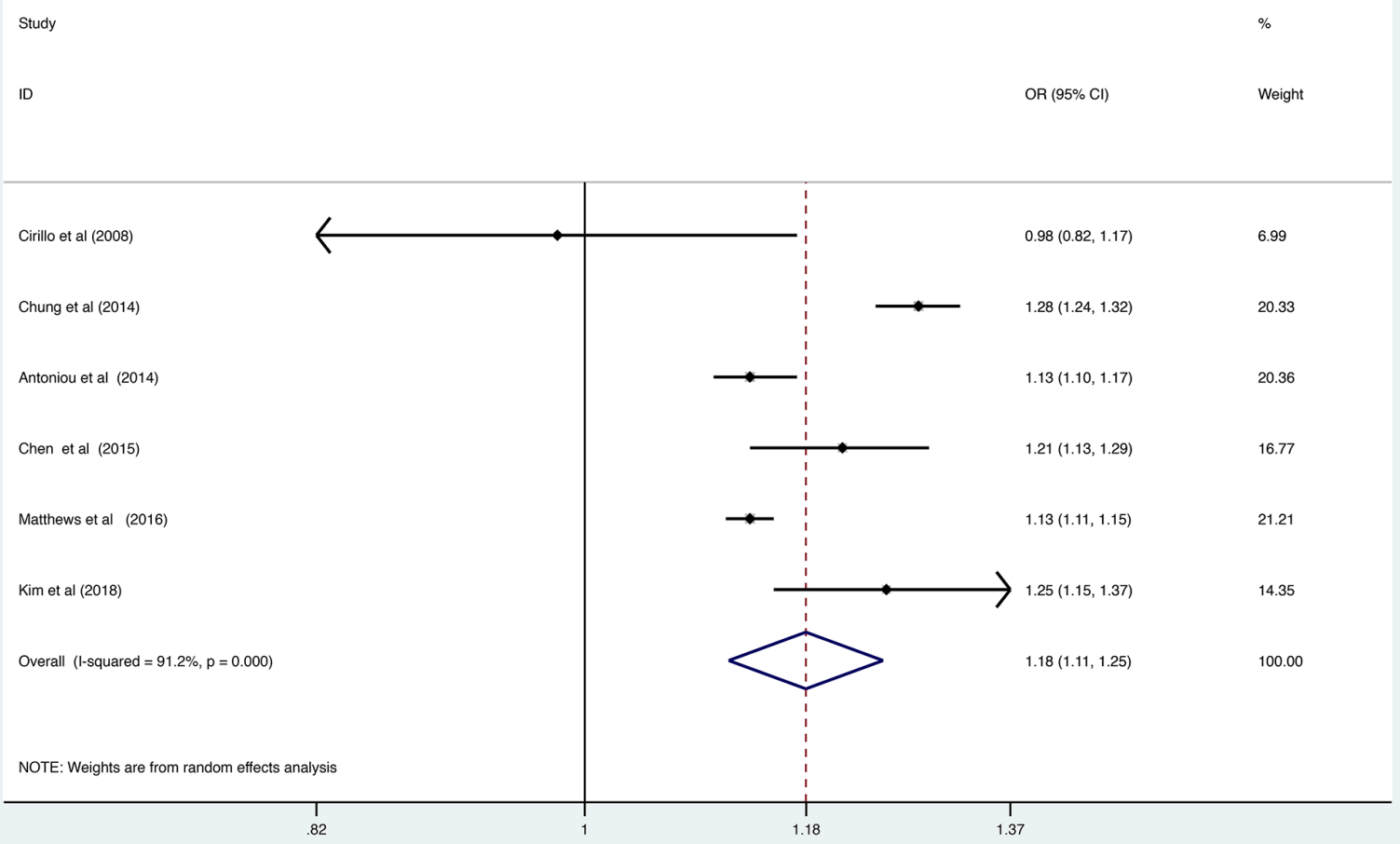

Figure 2 The squares and horizontal lines correspond to the study-specific OR and 95\% Cls. The area of the squares reflects the study-specific weight. Weights are from random-effect analysis. The diamond represents the pooled OR and $95 \% \mathrm{Cl}$. ID, identity.

number of study participants, participant mean age and the quality of study (table 2). The subgroup of study region (Asia: $\mathrm{I}^{2}=11 \%$, OR $1.26,95 \%$ CI 1.22 to 1.30; North America: $\mathrm{I}^{2}=58 \%$, OR $1.08,95 \%$ CI 0.95 to 1.23 ) exhibited marked decreases in heterogeneity. Therefore, we speculated that heterogeneity derives from regional differences. We found no association (OR $1.08,95 \%$ CI 0.95 to 1.23 ) between statin use and risk of herpes zoster in North America participants, but these results needed to be further verified due to the small number of studies in the different subgroups.

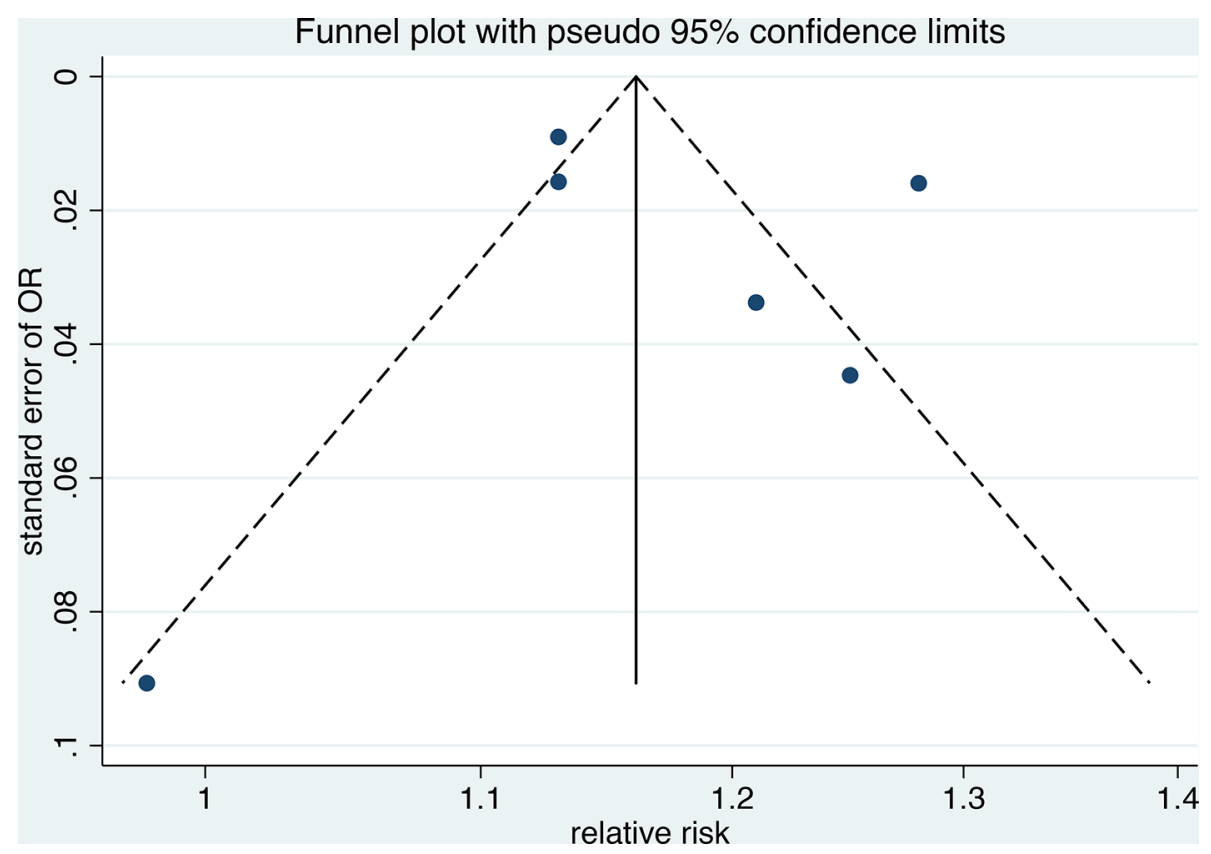

Figure 3 Circles represent identified studies. 
Table 2 Subgroup analyses of risk of herpes zoster among statin users

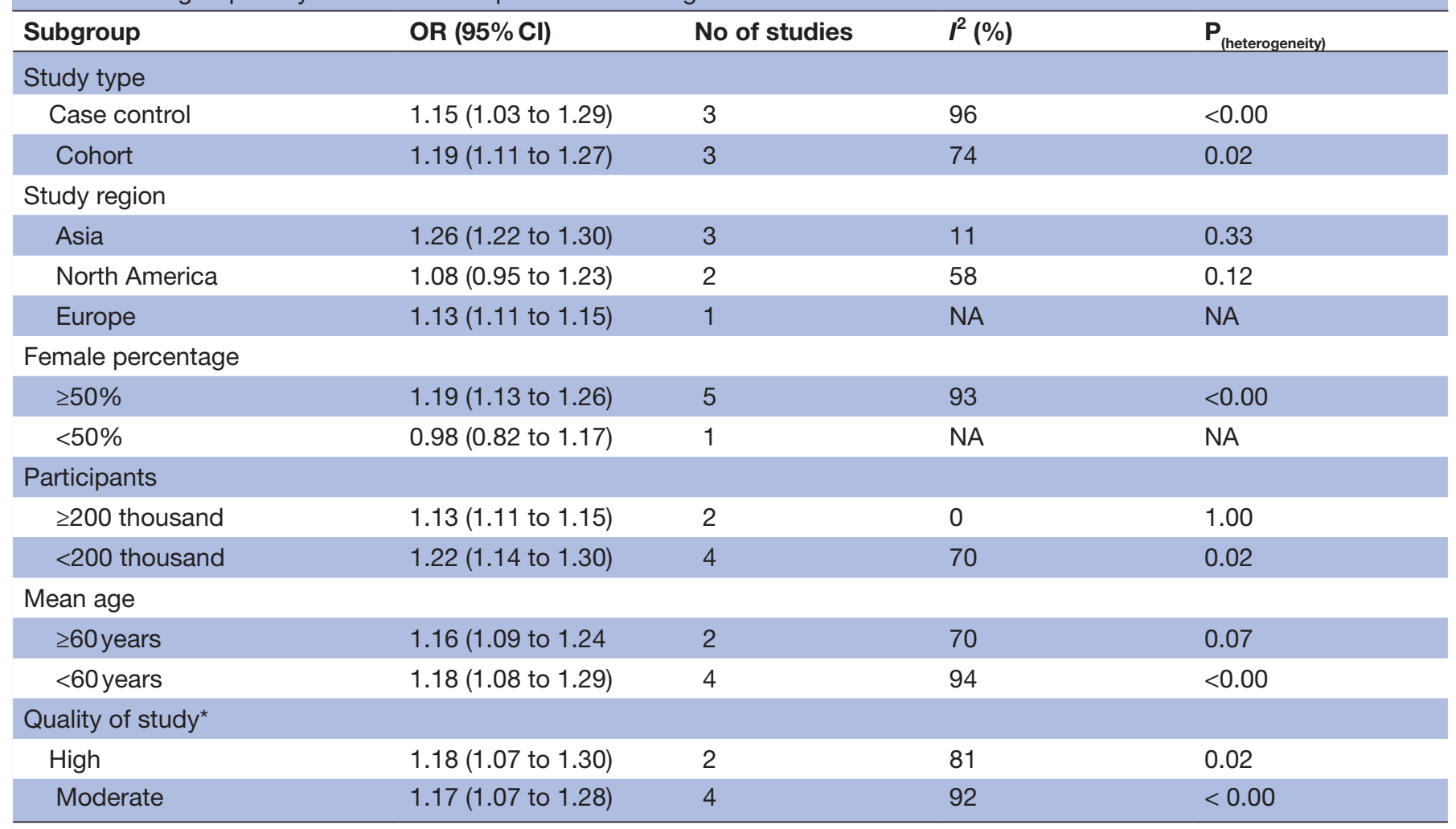

${ }^{*} \mathrm{~A}$ total score of 4-6 was considered moderate quality, and 7-9 was deemed high quality.

We performed a sensitivity analysis by sequentially omitting each study and then determining if the removal of the study led to a substantial change in the results. We found the study by Chung et al was a major source of heterogeneity (from $93 \%$ to $65 \%$ ). We omitted this study, performed the analysis again and found that the result was still significant (OR 1.15, 95\% CI 1.11 to 1.19).

\section{DISCUSSION}

In this meta-analysis of results from more than two million participants, we demonstrated a significantly increased risk of herpes zoster with an overall 1.18-fold increased risk among patients who used statins compared with non-users.

Stains can exhibit some immunomodulatory effects, including improving epithelial cell function, reducing oxidative stress and alleviating inflammation. ${ }^{30} 31$ Statins can impair T-cell activation and proliferation, ${ }^{3}{ }^{32}$ and statins can also decrease levels of some proinflammatory cytokines, ${ }^{33} 34$ potentially affecting the susceptibility to herpes zoster. One study showed that statins could increase the number of regulatory $\mathrm{T}$ cells (Tregs) in vivo, ${ }^{35}$ but a statin-induced Tregs increase may lead to the reactivation of latent viral infections, ${ }^{21}{ }^{36}$ like the VZV. Overall, the detailed mechanism of how statin use affects VZV remains unclear, and more studies are needed.

We typically associate the use of statins with hyperlipidaemia, but most studies did not assess serum cholesterol levels. This may lead to confounding by indication. Confounding by indication occurs if the effects of treatment indication are ignored, or if factors that may be a consequence of a condition are instead treated as potential causes of that condition. ${ }^{37} \mathrm{~A}$ higher dose or longer course of treatment suggests the presence of more serious hyperlipidaemia. Although Chen et $a l^{8}$ reported that younger statin users exhibited a higher risk of herpes zoster infection, the major risk factor for herpes zoster is increasing age ${ }^{38}{ }^{39}$ so the differing results may reflect insufficient adjustment or the consumption by younger patients of a high fat diet leading to high cholesterol level. Furthermore, one study with few participants found that cholesterol levels were associated with herpes zoster in cardiac transplant recipients. ${ }^{40}$ Although no mechanism has been established, serum cholesterol levels may be associated with the risk of herpes zoster. Statins are prescribed for both primary and secondary CVD, ${ }^{41}$ so it is unclear if the observed effects are due to the presence of CVD or the risk factors of CVD that increase herpes zoster risk rather than the use of statins. Although these studies tried to adjust for partial risk factors, additional studies are needed.

In our analysis, we found a potential contribution of gender to the risk of herpes zoster among statin users, though the effect was weak. Gender was previously identified as a risk factor of herpes zoster, ${ }^{38} 42$ and the use of statins might further increase the risk of infection. The 
gender difference for infection may indicate effects of sex hormones ${ }^{43}$ consistent with the ability of statins to regulate the oestrogen receptor. ${ }^{44}$ Recently, the use of statins was linked to the occurrence of diabetes ${ }^{45}$ for patients with no history of diabetes, and the incidence of herpes zoster has been reported to be much higher in patients with diabetes. ${ }^{46}{ }^{47}$ Because women are generally more susceptible to diabetes, these factors may be related. Again, more studies with a large number of participants are needed to confirm the result.

The strengths of our study include the following: we performed a comprehensive systematic search for eligible studies; this is the first systematic review and meta-analysis to specifically evaluate the association between statin use and the risk of herpes zoster; the studies included in our meta-analysis included many participants with long-term follow-up time; literature eligibility was assessed by two investigators independently; no significant publication bias was found.

Several limitations of this meta-analysis should be acknowledged. First, this was a meta-analysis of observational studies, allowing the conclusion that an association exists, but this type of analysis cannot determine if a causal relationship exists. Second, we found significant heterogeneity across studies, due to regional differences. Additionally, the study by Chung et al contributed much heterogeneity, but the result remained significant after the exclusion of this study. Third, we did not study the effects of different statins on the risk of herpes zoster due to insufficient data. Fourth, some results might be influenced by the conversion of other measures to OR. Overall, more studies are needed for more robust analysis.

\section{CONCLUSIONS}

Our meta-analysis indicates that the use of statins may increase the risk of herpes zoster. Because the studies included are limited and there is potential bias, further studies are warranted.

Acknowledgements The authors acknowledge that the Second Affiliated Hospital and Yuying Children's Hospital of Wenzhou Medical University for supporting the work of our study.

Contributors XG designed the study. LF and YW completed the extraction and analysis of data. LF and XL reviewed the results. LF wrote the report. All authors participated in the discussion and modification of the text. All authors approved the final version of the paper.

Funding The authors have not declared a specific grant for this research from any funding agency in the public, commercial or not-for-profit sectors.

Competing interests None declared.

Patient consent Not required.

Provenance and peer review Not commissioned; externally peer reviewed.

Data sharing statement All relevant study data can be found in the online supplementary files.

Open access This is an open access article distributed in accordance with the Creative Commons Attribution Non Commercial (CC BY-NC 4.0) license, which permits others to distribute, remix, adapt, build upon this work non-commercially, and license their derivative works on different terms, provided the original work is properly cited, appropriate credit is given, any changes made indicated, and the use is non-commercial. See: http://creativecommons.org/licenses/by-nc/4.0/.

\section{REFERENCES}

1. Johnston TP, Korolenko TA, Pirro M, et al. Preventing cardiovascular heart disease: Promising nutraceutical and non-nutraceutical treatments for cholesterol management. Pharmacol Res 2017;120:219-25.

2. Jasińska M, Owczarek J, Orszulak-Michalak D. Statins: a new insight into their mechanisms of action and consequent pleiotropic effects. Pharmacol Rep 2007;59:483.

3. Greenwood J, Steinman L, Zamvil SS. Statin therapy and autoimmune disease: from protein prenylation to immunomodulation. Nat Rev Immunol 2006;6:358-70.

4. Neuhaus O, Strasser-Fuchs S, Fazekas F, et al. Statins as immunomodulators: comparison with interferon-beta $1 \mathrm{~b}$ in MS. Neurology 2002;59:990-7.

5. Zoster EOH. Herpes zoster and postherpetic neuralgia. Essentials of Pain Medicine E-book 2011:358.

6. Schmader K. Herpes Zoster. Clin Geriatr Med 2016;32:539-53.

7. Antoniou $\mathrm{T}$, Zheng $\mathrm{H}$, Singh $\mathrm{S}$, et al. Statins and the risk of herpes zoster: a population-based cohort study. Clin Infect Dis 2014;58:350-6.

8. Chen $\mathrm{HH}$, Lin CL, Yeh CJ, et al. Statins can increase the risk of herpes zoster infection in Asia. Eur J Clin Microbiol Infect Dis 2015;34:1451-8.

9. Chung SD, Tsai MC, Liu SP, et al. Herpes zoster is associated with prior statin use: a population-based case-control study. PLoS One 2014;9:e111268.

10. Cirillo DJ. The effect of statin use on incident immune-mediated and infectious conditions among US. veterans. 2008. Dissertations \& Theses - Gradworks.

11. Kim MC, Yun SC, Lee SO, et al. Statins increase the risk of herpes zoster: a propensity score-matched analysis. PLoS One 2018;13:e0198263.

12. Matthews A, Turkson M, Forbes $\mathrm{H}$, et al. Statin use and the risk of herpes zoster: a nested case-control study using primary care data from the U.K. Clinical Research Practice Datalink. Br J Dermatol 2016;175:1183-94

13. Stroup DF, Berlin JA, Morton SC, et al. Meta-analysis of observational studies in epidemiology: a proposal for reporting. Meta-analysis Of Observational Studies in Epidemiology (MOOSE) group. JAMA 2000;283:2008-12.

14. Stang A. Critical evaluation of the Newcastle-Ottawa scale for the assessment of the quality of nonrandomized studies in metaanalyses. Eur J Epidemiol 2010;25:603-5.

15. Margulis AV, Pladevall M, Riera-Guardia N, et al. Quality assessment of observational studies in a drug-safety systematic review, comparison of two tools: the Newcastle-Ottawa Scale and the RTI item bank. Clin Epidemiol 2014;6:359.

16. Higgins JP, Thompson SG, Deeks JJ, et al. Measuring inconsistency in meta-analyses. BMJ 2003;327:557-60.

17. DerSimonian R, Laird N. Meta-analysis in clinical trials. Control Clin Trials 1986;7:177-88.

18. Wang J, Shen Y, Wang J, et al. Relation of phosphodiesterase type 5 inhibitors and malignant melanoma: a meta-analysis and systematic review. Oncotarget 2017;8:46461-7.

19. Egger M, Davey Smith G, Schneider M, et al. Bias in meta-analysis detected by a simple, graphical test. BMJ 1997;315:629-34.

20. Huang S, Yang Z, Ma Y, et al. miR-101 Enhances Cisplatin-induced DNA damage through decreasing Nicotinamide Adenine Dinucleotide Phosphate levels by directly repressing Tp53-induced glycolysis and apoptosis regulator expression in prostate cancer cells. DNA Cell Biol 2017;36:303-10.

21. Goldstein MR, Mascitelli L, Pezzetta F. The double-edged sword of statin immunomodulation. Int J Cardiol 2009;135:128-30.

22. Kalra S, Chawla A. Herpes zoster and diabetes. J Pak Med Assoc 2016;66:1042-3.

23. Pirmohamed M. Statins, immunomodulation, and infections: a complex and unresolved relationship. Clin Infect Dis 2014:58:357-8.

24. Shalom G, Cohen AD. Statin exposure and the risk for herpes zoster: implications for public health. Br J Dermatol 2016;175:1137-8.

25. Yuet WC, Khine H, Ahmad Z. Statin-associated adverse events. Clinical Medicine Insights: Therapeutics 2015;7:CMT.S18865-24.

26. McKinney WP, Horowitz MM, Battiola RJ. Susceptibility of hospitalbased health care personnel to varicella-zoster virus infections. Am J Infect Control 1989;17:26-30.

27. Terao M, Yamamoto T, Umeda J, et al. Drug induced herpes zoster (Do statin induce herpes zoster?). Skin Research 2005;4:335-8. 
28. Cirillo DJ, Wallace RB. Statin use on incident immune-mediated and infectious conditions in the veterans administration health system. Journal of General Internal Medicine 2012;27:S309-S10.

29. Antoniou T, Juurlink DN, Mamdani MM, et al. Reply to Strandberg and Tienari. Clin Infect Dis 2014;58:1043-4.

30. Laufs U, Liao JK. Isoprenoid metabolism and the pleiotropic effects of statins. Curr Atheroscler Rep 2003;5:372-8.

31. Liao JK, Laufs U. Pleiotropic effects of statins. Annu Rev Pharmacol Toxicol 2005;45:89-118.

32. Ghittoni R, Lazzerini PE, Pasini FL, et al. T lymphocytes as targets of statins: molecular mechanisms and therapeutic perspectives. Inflamm Allergy Drug Targets 2007;6:3-16.

33. Inhibition of proinflammatory cytokine production by pravastatin. The Lancet.

34. Rezaie-Majd A, Maca T, Bucek RA, et al. Simvastatin reduces expression of cytokines interleukin-6, interleukin-8, and monocyte chemoattractant protein-1 in circulating monocytes from hypercholesterolemic patients. Arterioscler Thromb Vasc Biol 2002;22:1194-9.

35. Mausner-Fainberg K, Luboshits G, Mor A, et al. The effect of HMGCoA reductase inhibitors on naturally occurring CD4+CD25+ T cells. Atherosclerosis 2008;197:829-39.

36. Belkaid Y, Rouse BT. Natural regulatory $T$ cells in infectious disease. Nat Immunol 2005;6:353-60.

37. Psaty BM, Koepsell TD, Lin D, et al. Assessment and control for confounding by indication in observational studies. J Am Geriatr Soc 1999;47:749-54
38. Amicizia D, Domnich A, Arata L, et al. The role of age-sex interaction in the development of post-herpetic neuralgia. Hum Vaccin Immunother 2017;13:376-8.

39. Blum A. HMG-CoA reductase inhibitors (statins), inflammation, and endothelial progenitor cells-New mechanistic insights of atherosclerosis. Biofactors 2014;40:295-302.

40. Del Pozo JL, van de Beek D, Mandrekar JN, et al. High serum cholesterol levels are associated with herpes zoster infection after heart transplantation. Clin Infect Dis 2010;50:121-2.

41. Care N. Drug therapy for the primary prevention of cardiovascular disease (CVD): Royal College of General Practitioners (UK), 2008.

42. Opstelten W, Van Essen GA, Schellevis F, et al. Gender as an independent risk factor for herpes zoster: a population-based prospective study. Ann Epidemiol 2006;16:692-5.

43. McClelland EE, Smith JM. Gender Specific Differences in the Immune Response to Infection. Arch Immunol Ther Exp 2011;59:203-13.

44. Taylor HS, Alderman lii M, D'Hooghe TM, et al. Effect of simvastatin on baboon endometriosis. Biol Reprod 2017;97:32-8.

45. Ridker PM, Pradhan A, Macfadyen JG, et al. Cardiovascular Benefits and Diabetes Risks of Statin Therapy in Primary Prevention. Lancet 2012;56:565.

46. Aldaz P, Díaz JA, Loayssa JR, et al. [Herpes zoster incidence in diabetic patients]. An Sist Sanit Navar 2013;36:57-62.

47. Guignard AP, Greenberg M, Lu C, et al. Risk of herpes zoster among diabetics: a matched cohort study in a US insurance claim database before introduction of vaccination, 1997-2006. Infection 2014;42:729-35. 